\section{The buzzer as a primary aversive stimulus: II. Unavoidable buzzer presentations and conditioned acceleration}

\author{
DAVE RIESS \\ Galesburg State Research Hospital, Galesburg, Ill. 61401
}

Two experiments explored the effects of a $76-\mathrm{dB}$ buzzer on Sidman avoidance. In Experiment 1, it was shown that a VI-1 schedule of unavoidable 2-sec buzzer presentations would increase avoidance rates during entire 30 -min sessions. In Experiment 2 it was shown that conditioned acceleration could be produced when a light CS predicted a $5-\mathrm{sec}$ buzzer UCS. Both these effects were small in magnitude but highly reliable, and both effects attenuated rapidly when the buzzer was removed.
Previous research (Riess, 1970a) indicated that a $76-\mathrm{dB}$ buzzer increase response rates during 1-min presentations ("unconditioned" acceleration) and that the normal increases in avoidance rates which can be elicited by a CS previously paired with shock (conditioned acceleration) can be increased still further when the CS is presented in compound with a buzzer (summation). The present studies are the third and fourth of a series of 14 experiments designed to explore the unconditioned aversive properties of buzzers in the major paradigms of aversive control and are essentially replications of two phenomena obtained with shocks and first reported by Sidman, Herrnstein, \& Conrad (1957). In these studies it was shown that (1) periodic unavoidable shocks delivered independently of ongoing avoidance behavior will produce increases in responding and (2) a CS that predicts the occurrence of such an unavoidable shock will also elicit increases in avoidance responding. The first of the present two experiments explores the effects of "free" buzze r presentations on a Sidman shock-avoidance baseline and the second attempts to determine if the unconditioned (Riess, 1970a) are conditionable (i.e., if a CS predicts a buzzer occurrence, will the CS itself eventually elicit the increases?). EXPERIMENT 1:

UNCONDITIONED ACCELERATION WITH INESCAPABLE BUZZER PRESENTATIONS Subjects

The Ss were three Wistar female rats who were raised in an enriched environment described previously (Riess \& Plaut, 1970). They had a history of avoidance (Riess, 1970b), long enough to insure the terminal response rates required for the present experiment, but were naive with respect to buzzers. superimposed on Sidman avoidance would effects of buzzers previously reported
(4) Baseline reversal was identical to Step 2 and was continued for six sessions. (5) Re-presentation was identical to Step 3 and lasted two sessions. (6) Baseline was identical to Step 2 and lasted for four additional sessions.

Results and Discussion

The results in Fig. 1 may be considered an ABABA design, with the open figures representing the baseline and the closed figures representing the effects of the VI-1 buzzer schedule.

The average response rate was around four responses per minute in the absence of the buzzer and around five responses per minute during the buzzer schedule, and all of the reversals produced abrupt shifts in rate toward the average value for the prevailing experimental condition, although there is some suggestion that each successive withdrawal of the buzzer schedule produced a slightly greater delay in the return to the original baseline. EXPERIMENT 2:

\section{CONDITIONED ACCELERATION} WITH A BUZZER UCS

Subjects and Apparatus

The Ss were four naive female Wistar albinos taken from the colony maintained by the Galesburg State Research Hospital Psychology Laboratory. They were between 72 and 82 days old at the beginning of the experiment. Apparatus was identical to that of Experiment 1.

\section{Procedure}

Ss were run through a four-step sequence consisting of the following:

(1) Hurdle cross acquisition consisted of nine daily 30-min sessions of Sidman avoidance at $\mathrm{RS}=20, \mathrm{SS}=5$, and shocks $=$ $.15 \mathrm{sec}, 1 \mathrm{~mA}$.

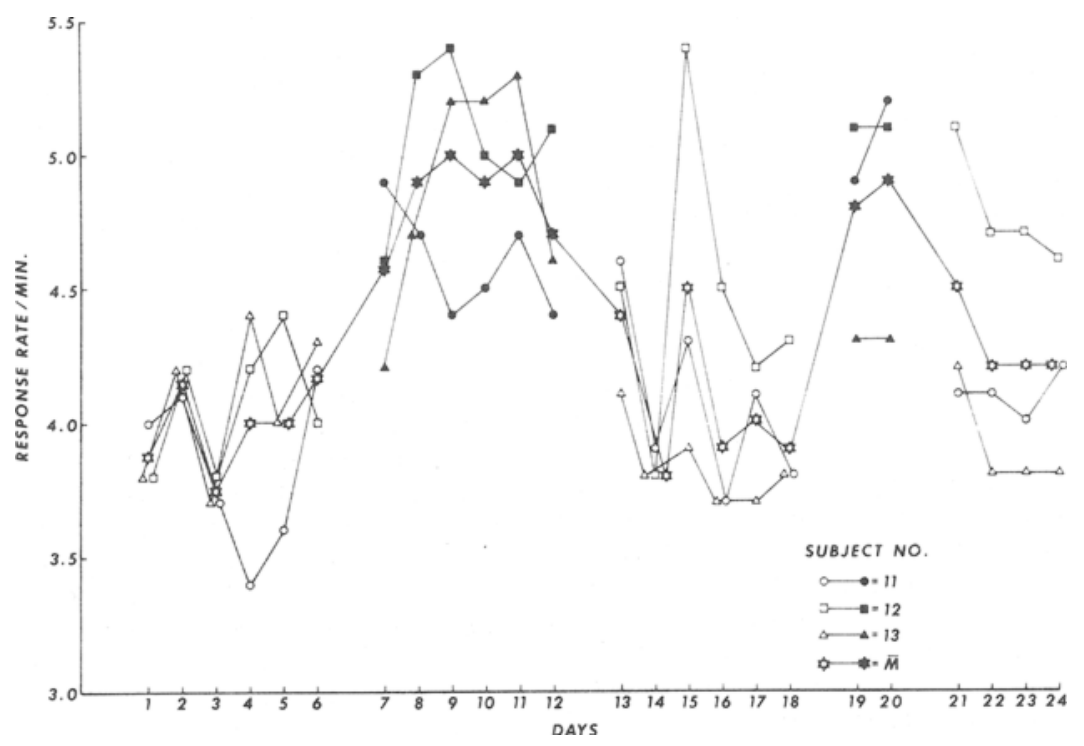

Fig. 1. Sidman avoidance rates during a VI-1 schedule of buzzer presentations (dark) and without the buzzer (light). 


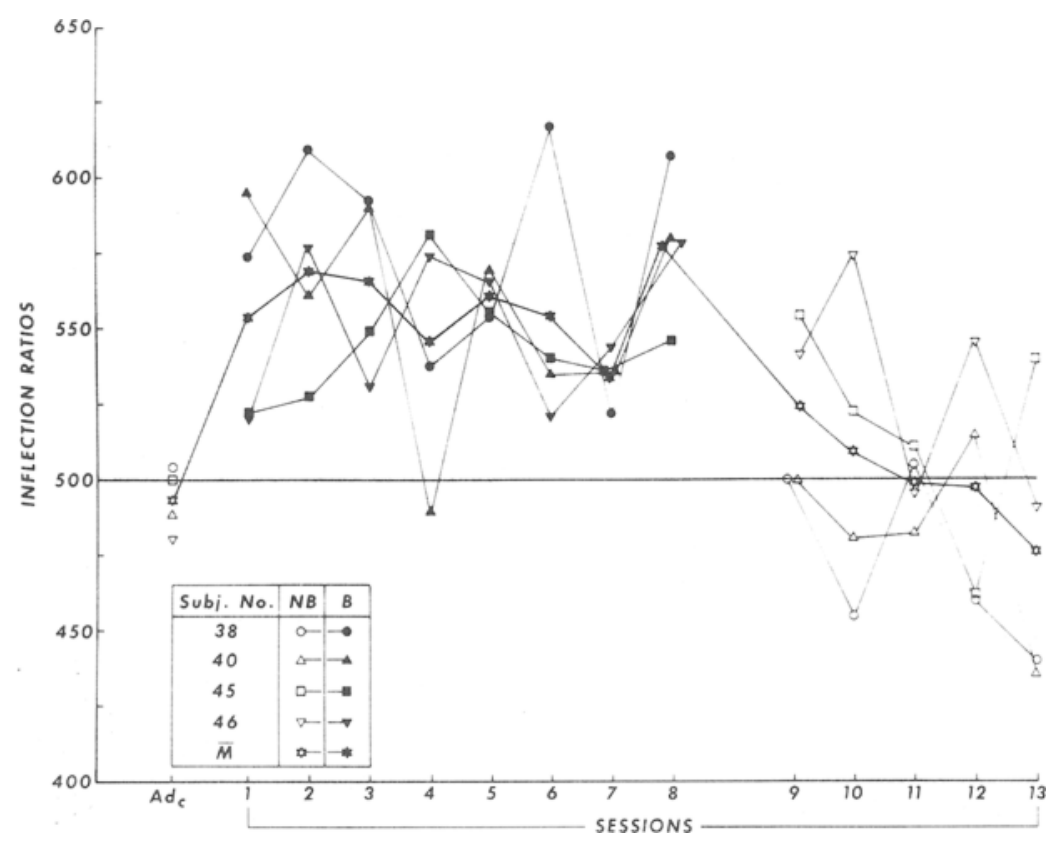

Fig. 2. Avoidance rate changes during a light CS presented alone (Adc), reinforced with a 5-sec buzzer UCS (dark), and again without the buzzer (NB) during extinction. A ratio of .500 indicates no change in rate and .600 indicates a $50 \%$ increase in rate.

(2) CS adaptation consisted of five $30-$ sec red-light presentations at the start of the 10th, 15th, 20th, 25th, and 30th min of the avoidance sessions. A rate-change ratio was computed from the formula CIR $=2 B /(A+2 B)$, where $C I R=$ conditioned inflection ratio, $\mathrm{A}=$ avoidance responses during 1 -min preceding $\mathrm{CS}$ onset, and $\mathrm{B}=$ avoidance responses during the red light. Adaptation was continued until each $\mathrm{S}$ met a criterion of two consecutive sessions with the CIR average for all 10 presentations between .475 and .525 . In the event some Ss reached criterion before others, they were continued at Step 1, so the amount of avoidance would be identical for all Ss.

(3) Pavlovian conditioning was identical to Step 2 except that 5 -sec buzzer presentations occurred at the end of the light presentations. The light period was extended $5 \mathrm{sec}$, making the $C S$ period equal to $35 \mathrm{sec}$ and the paradigm coterminous delay conditioning. Responding during the UCS was ignored in computing the CIR. This was continued for eight sessions.

(4) Pavlovian extinction was identical to Step 2, thus completing the third leg of the ABA design. This was continued until the mean inflection ratio was below .500 for three successive sessions.

Results and Discussion

The results are presented in Fig. 2, which may be considered an ABA design with the first $A$ (adaptation) and the second A (extinction) representing the baselines and the closed figures normally it involves a decrease from above .500 toward criterion. The fact that extinction did not stop at .500 but continued to drop also supports this interpretation. The drop to about .430 in Ss 38 and 40 in Session 13 must be considered quite unusual in view of the fact that the shock contingencies were still in effect during CS presentations and because normal avoidance in the shuttlebox is quite efficient (Riess, 1970c), with even slight decreases in response rate running a high risk of producing more shocks. Were this tendency not present, the ratios during conditioning would probably be more comparable to those obtained with shock, as shown by LoLordo (1967) in the case of a loud noise UCS. Brody (1966) has also produced levels of conditioned suppression comparable to those obtained with shock using a loud noise.

The present results for both experiments are in basic agreement with those of Sidman et al (1957), using unavoidable shocks in ways analogous to the use of the buzzer in the present two studies. The most parsimonious interpretation of the present results would seem to be that a buzzer of sufficient intensity is a primary unconditioned aversive stimulus, and therefore any phenomena obtainable with noxious shock stimuli should be essentially replicable with buzzers. This hypothesis has already been confirmed with escape behavior (Myers, 1965), self-punitive behavior (Melvin \& Martin, 1966), and unconditioned acceleration (Riess, 1970a) and is to receive further testing in this laboratory in the avoidance, punishment, and conditioned-suppression pafadigms. effect another adaptation trial, and the asymptotic acceleration levels are therefore reached in Session 1. Similarly, during extinction, only 10 unreinforced CS presentations (Sessions 9 and 10) are required to bring the inflection ratios back to below .500 . Both the rapid acquisition and extinction are probably related to the fact that the UCS was of a different sensory modality than the aversive event in the avoidance schedule, and the discrimination of the presence or absence of the UCS therefore developed more quickly than it would have if the CS had predicted an unavoidable shock. The magnitude of the acceleration here is somewhat less than that characteristically obtained with a shock UCS; and although it is tempting to conclude that this is because the buzzer is a less aversive stimulus than a shock, some consideration must be given also to the strong tendency of the CS used here to elicit suppression. Adaptation proved largely a matter of waiting for the inflection ratios to increase from their initial levels to .500 , whereas

\section{REFERENCES}

BRODY, J. F, Conditioned suppression maintained by loud noise instead of shock. Psychonomic Science, 1966, 6, 27-28.

LoLORDO, V. M. Similarity of conditioned fear responses based upon different aversive events. Joumal of Comparative \& Physiological Psychology, 1967, 64, 154-158.

MELVIN, K. B., \& MARTIN, R. C. Facilitative effects of two modes of punishment on resistance to extinction. Journal of Comparative \& Physiological Psychology, $1966,62,491-494$.

MEYERS, A. K. Instrumental escape conditioning to a low-intensity noise by rats. Journal of Comparative \& Physiological Psychology, 1965, 60, 82-87.

RIESS, D. The buzzer as a primary aversive stimulus: I. Unconditioned acceleration and summation of conditioned and unconditioned acceleration. Psychonomic Science, 1970, 21 167-169.

RIESS, D. Pavlovian phenomena in conditioned acceleration: Discrimination. Manuscript available from the Psychology Department, Galesburg (IIl.) State Research Hospital, 1970b.

RIESS, D. Shuttleboxes, Skinner boxes, and Sidman avoidance: Acquisition and terminal 
performance as a function of response topography. Manuscript available from the Psychology Department, Galesburg (Ill.) State Research Hospital, 1970c.

RIESS. D., \& BATH, J. Operant and Pavlovian stimulus control of avoidance latencies in conditioned acceleration. Psychonomic Science, 1970, 19, 165-167.

RIESS, D., \& PLAUT, S. M. Sidman avoidance acquisition as a function of se alle enrlched environment. Manuseript available trom the Psychology Department. Galesburg (Ill.) State Research Hospital. 1970.

SIDMAN, II.. HERRNSTEIN, R. J.. \& CONRAD. D. G. Maintenance of avoidance behavior by unavoidable shocks. Journal of Comparative \& Physiological Psychology, $1957,50,553-557$

\title{
Shock-avoidance and shock-escape drinking in rats: Rate of licking*
}

\author{
M. L. J. CRAWFORD \\ Baylor College of Medicine \\ and The University of Texas Graduate School of Biomedical Sciences \\ Houston, Tex. 77025
}

The drinking rates in rats were measured by interresponse times for licking under shock-avoidance or shock-escape contingent licking conditions. Interresponse times were quite comparable under either set of shock-contingent drinking conditions with the modal interlick interval approximately $145 \mathrm{msec}$. Well-established principles of behavior maintained on fixed ratio schedules would predict such results.

Stellar \& Hill (1952) and Corbit \& Luschei (1969) have described the invariant nature of the rate of drinking for water in the water-deprived rat and where the solution being consumed incident to the licking could be considered to be preferred or aversive in taste stimulation. The interresponse lick rate (IRT) did not change, even though hours of water deprivation and wide saccharin concentrations produced predictable changes in the volumes of solution ingested within a short test period, the volume of solution ingested being a function of the time spent in drinking.

Data reported here support the conclusions of the above researchers by extending the boundary conditions under which the drinking behavior is stable in that the consequence of the licking behavior may be (1) the avoidance of shock to the feet, (2) the escape from a repeated shock to the feet, and/or (3) the avoidance of, or escape from, a discriminative stimulus (SD) that has been associated with the occurrence of shock for a large number of trials.

\section{METHOD}

Data were collected for periods up to 20 months from six rats, albino and hooded,

*This research was supported in part from a NIH research grant (MN 14434-01) to the Texas Research Institute for Mental Sciences.

\section{of both sexes.}

An experimental cage was provided with a drinking tube, accessible to the rat through a hole in the cage wall at one end. The tube permitted the rat's tongue to complete an electrical contact with the sensing apparatus only through the drinking solution. Such contacts between the drinking tube and the grid floor of the cage were detected by a Grason-Stadler Drinkometer. The floor was electrically common to the lick detection system and to a scrambled shock source (SDS) through a relay bank that prevented shock delivery to the rat's tongue. The experimental cage was enclosed in a large ventilated sound-attenuated chamber having an overhead 25-W house lamp that served as a discriminative stimulus (SDL) and was controlled remotely. A digital logic system controlled program requirements and supplied classified lick responses, relative to the SD, to the input of a TMC Computer of Average Transients (C.A.T. 1000) for histogramming of interlick intervals (IRTS). Cumulative records were also taken.

\section{PROCEDURE}

The rats were initially deprived of water for $23 \mathrm{~h}$ and given $1 \mathrm{~h}$ training with the termination of the house light contingent upon licking on a CRF schedule. After three to four such sessions, shock was introduced and increased in intensity until avoidance motor response of the rat was obvious to the E. Shock duration was $0.5 \mathrm{sec}$ and did not occur when the light was off. During subsequent training sessions the $\mathrm{Ss}$ were not under deprivation, always having food and water freely available in the home cage.

The rat was removed from the home cage, weighed, and placed in the experimental chamber with the house light (SDL) off. For a variable period up to $30 \mathrm{~min}$, the chamber remained dark, and any drinking was recorded. At the end of this period, the SDL came on and remained on for $20 \mathrm{sec}$, unless the rat emitted up to nine licks on the drinking tube, turning off the light. The light remained off for $30 \mathrm{sec}$ and the cycle was repeated, unless the $S$ emitted four licks during this period, which kept the light turned off. However, if the FR9 ratio was not met within the first $20 \mathrm{sec}$ following onset of the light, a brief $(0.5-\mathrm{sec})$ shock was delivered to the feet, recurring every $6 \mathrm{sec}$, while the light remained on. To escape the recurrent shock, the rat had to emit 20 licks, after which the cyclic shock and light-on period terminated and the entire cycle was repeated after a $30-\mathrm{sec}$ interval. Therefore, the schedule was a FR4 FR9FR20 sequential schedule. Time-out periods of 15-20 min, where the house light was off and no shocks occurred, followed 20- to 30 -min sessions where avoidance of shock was contingent upon licking. The logic system separated the licking responses, and counters tallied those responses occurring (1) during light off, (2) light on but before first shock, (3) after first shock, (4) total responses. Responses with in these separated response classes were measured for interresponse times (IRTs).

At the end of the experimental session, the amount of liquid consumed during the session was measured. The present data, collected from six rats, were obtained where the rat licked solutions of either $10 \%$ or $20 \%$ ethanol in a commercially available breakfast drink (Tang; $70 \mathrm{~g} / 500 \mathrm{cc}$ solution).

\section{RESULTS}

As the rate of drinking was quite comparable for all six Ss (modal response $140 \pm 10 \mathrm{msec}$ under all conditions), data from only two $S s$ are presented in Fig. 1. Figure 1A demonstrates the degree of discrimination of the SDL and SDS in that the rat emitted no responses in the 20-min period prior to the occurrence of the first SDL and SDS sequence. After a brief "warm-up" the efficiency in terminating the SDL and consequently avoiding the SDS became very high. During the two long "runs" of licking in the first segment of the session, 200 IRTs were 